\title{
Generalized Langevin equations: Anomalous diffusion and probability distributions
}

\author{
Josep M. Porrà, Ke-Gang Wang, and Jaume Masoliver \\ Departament de Física Fonamental, Universitat de Barcelona, Diagonal 647, 08028 Barcelona, Spain
}

(Received 16 October 1995; revised manuscript received 27 November 1995)

\begin{abstract}
We study the motion of a particle governed by a generalized Langevin equation. We show that, when no fluctuation-dissipation relation holds, the long-time behavior of the particle may be from stationary to superdiffusive, along with subdiffusive and diffusive. When the random force is Gaussian, we derive the exact equations for the joint and marginal probability density functions for the position and velocity of the particle and find their solutions. [S1063-651X(96)10706-6]
\end{abstract}

PACS number(s): 02.50.-r, 05.40.+j, 05.60.+w, 05.20.Dd

\section{INTRODUCTION}

This paper deals with systems that present anomalous diffusion. It has been shown that in many systems the mean square displacement does not grow linearly as in normal diffusion but in the anomalous form given by

$$
\left\langle X^{2}(t)\right\rangle \sim t^{\nu} \quad(t \rightarrow \infty),
$$

where $\nu \neq 1[1,2]$. There is current interest in understanding the physical mechanism leading to anomalous diffusion and it is thus found that chaotic systems can present superdiffusion with $\nu>1$ [3] while subdiffusion with $\nu<1$ is encountered in constrained systems such as fractals $[1,2]$. We have recently shown that the consideration of inertial effects also results in superdiffusion [4-6]. Moreover, we have also observed that Gaussian $1 / f$ driving noise leads to a subdiffusive behavior for the undamped free Brownian particle $[7,8]$.

On the other hand, generalized Langevin equations have been used recently to describe the dynamics of particles in percolation clusters that present anomalous diffusion [9]. This equation is a nonlocal equation that, in the absence of a deterministic field, can be written in the form

$$
\ddot{X}(t)+\int_{0}^{t} \beta\left(t-t^{\prime}\right) \dot{X}\left(t^{\prime}\right) d t^{\prime}=F(t),
$$

where $\beta\left(t-t^{\prime}\right)$ is the dissipative memory kernel and $F(t)$ is a random force that we assume to be zero centered and stationary, i.e.,

$$
\left\langle F(t) F\left(t^{\prime}\right)\right\rangle=C\left(\left|t-t^{\prime}\right|\right)=C(\tau),
$$

where $C(\tau)$ is the correlation function $[10,11]$. When the system described by Eq. (1.1) is in the equilibrium state, then the functions $\beta(t)$ and $C(t)$ are related to each other by means of the fluctuation-dissipation theorem [11]:

$$
C(t)=k_{B} T \beta(t),
$$

where $k_{B}$ is the Boltzmann constant and $T$ is the absolute temperature of the environment (heat bath). In this case the random force is sometimes referred to as "internal noise." Note that, from a physical point of view, one of the effects of the noise being internal is that the relaxation time of the system is essentially the same as the correlation time of the noise. However, in nonequilibrium systems the driving noise and the dissipation may have different origin and no fluctuation-dissipation relation holds. In such a case $F(t)$ will be referred to as "external noise."

In this paper, we will show how the long-time behavior of the mean square displacement for systems described by generalized Langevin equations (GLE's) depends on the properties of the correlation function and of the memory kernel. We consider nonequilibrium systems although the equilibrium situation is easily recovered by imposing fluctuationdissipation relation. When both the correlation function and the dissipative kernel are long-time tail functions, a variety of limiting behaviors is obtained. The exponents of the longtime tails determine whether the system becomes stationary, subdiffusive, diffusive, superdiffusive, or even logarithmic, i.e., $\left\langle X^{2}(t)\right\rangle \sim \ln t$.

When the noise is Gaussian, and due to the linearity of Eq. (1.1), it is also possible to derive exact expressions and Fokker-Planck equations for the joint probability density, $p(x, v, t)$, of the displacement and the velocity and for the marginal densities $p(x, t)$ and $p(v, t)$.

The paper is organized as follows. In Sec. II, we set the general analysis of the process governed by Eq. (1.2). In Sec. III we study the long-time behavior of the variances and discuss the anomalous diffusion. In Sec. IV we analyze two relevant examples. In Sec. V we obtain the probability density functions of the process when the driving noise is Gaussian. Conclusions are drawn in Sec. VI and technical aspects are in the Appendices.

\section{GENERAL ANALYSIS}

In what follows we will assume that the driving noise $F(t)$ is stationary, zero centered, and has a correlation function given by $C(t)$. We write the formal solution to Eq. (1.1) in the form

$$
X(t)=\langle X(t)\rangle+\int_{0}^{t} H(t-\tau) F(\tau) d \tau
$$

where

$$
\langle X(t)\rangle=x_{0}+v_{0} H(t),
$$


where $x_{0}=X(0)$ and $v_{0}=\dot{X}(0)$ are the initial position and the initial velocity of the particle. The relaxation function $H(t)$ is the inverse form of the Laplace transform

$$
\hat{H}(s)=\frac{1}{s^{2}+s \hat{\beta}(s)},
$$

where

$$
\hat{\beta}(s)=\int_{0}^{\infty} \beta(t) e^{-s t} d t
$$

is the Laplace transform of the dissipative memory kernel. The time derivative of Eq. (2.1) yields

$$
\dot{X}(t)=\langle\dot{X}(t)\rangle+\int_{0}^{t} h(t-\tau) F(\tau) d \tau
$$

where $\langle\dot{X}(t)\rangle=v_{0} h(t)$ and the relaxation function $h(t)$ is the derivative of $H(t)$, i.e., $h(t)=\dot{H}(t)$. Hence,

$$
\hat{h}(s)=\frac{1}{s+\hat{\beta}(s)} .
$$

In addition, from Eqs. (2.2) and (2.5) it follows that $H(0)=0$ and $h(0)=1$.

We will only consider random systems whose velocity relaxes to a stationary state with zero average velocity. Thus, $h(t)$ goes to zero when $t \rightarrow \infty$. For systems driven by internal noise the relaxation function $h(t)$ is related with the longtime behavior of the autocorrelation function of the velocity. This relation is proved in Appendix A and reads

$$
h(\tau)=\lim _{t \rightarrow \infty} \frac{\langle\dot{X}(t+\tau) \dot{X}(t)\rangle}{\langle\dot{X}(t) \dot{X}(t)\rangle} .
$$

From Eqs. (2.1) and (2.5) and the symmetry property of the correlation function, i.e., $C\left(t-t^{\prime}\right)=C\left(t^{\prime}-t\right)$, we obtain the explicit expression of the variances of process (1.1)

$$
\begin{aligned}
\sigma_{x x}^{2}(t) & \equiv\left\langle[X(t)-\langle X(t)\rangle]^{2}\right\rangle \\
& =2 \int_{0}^{t} H\left(t_{1}\right) d t_{1} \int_{0}^{t_{1}} H\left(t_{2}\right) C\left(t_{1}-t_{2}\right) d t_{2}, \\
\sigma_{v v}^{2}(t) & \equiv\left\langle[\dot{X}(t)-\langle\dot{X}(t)\rangle]^{2}\right\rangle \\
& =2 \int_{0}^{t} h\left(t_{1}\right) d t_{1} \int_{0}^{t_{1}} h\left(t_{2}\right) C\left(t_{1}-t_{2}\right) d t_{2},
\end{aligned}
$$

and

$$
\begin{aligned}
\sigma_{x v}^{2}(t) & \equiv\langle[X(t)-\langle X(t)\rangle][\dot{X}(t)-\langle\dot{X}(t)\rangle]\rangle=\frac{1}{2} \dot{\sigma}_{x x}^{2}(t) \\
& =\int_{0}^{t} H\left(t_{1}\right) d t_{1} \int_{0}^{t} h\left(t_{2}\right) C\left(t_{1}-t_{2}\right) d t_{2} .
\end{aligned}
$$

These are general equations valid for either internal and external noise. However, when the driving noise is internal Eqs. (2.8)-(2.10) reduce to the following more convenient form [12]:

$$
\begin{gathered}
\sigma_{x x}^{2}(t)=k_{B} T\left[2 \int_{0}^{t} H\left(t^{\prime}\right) d t^{\prime}-H^{2}(t)\right], \\
\sigma_{v v}^{2}(t)=k_{B} T\left[1-h^{2}(t)\right],
\end{gathered}
$$

and

$$
\sigma_{x v}^{2}(t)=k_{B} T H(t)[1-h(t)] .
$$

\section{ASYMPTOTIC BEHAVIOR AND ANOMALOUS DIFFUSION}

We will now discuss with some generality the asymptotic behavior of the mean square displacement in terms of the asymptotic expansions of the correlation function $C(t)$ and of the memory kernel $\beta(t)$.

We will not study all possible limiting behaviors of the correlation function. Instead we will only consider two simple but very general models, which are described below. In both cases we will assume that the correlation function is a locally integrable function in $(0, M)$ for any positive $M$.

(a) Finite noise intensity. In this case the noise intensity defined by [13]

$$
K \equiv \int_{0}^{\infty} C(t) d t \neq 0
$$

is finite and nonvanishing. Hence, $C(\tau)=o\left(\tau^{-1}\right)$ when $\tau \rightarrow \infty$. Once we know the leading behavior of $C(\tau)$ as $\tau \rightarrow \infty$, the behavior of its Laplace transform $\hat{C}(s)$ as $s \rightarrow 0$ can be obtained using Abelian theorems, which give the small $s$ behavior of $\hat{C}(s)$ if one knows the asymptotic behavior of $C(\tau)$ as $\tau \rightarrow \infty$ [14]. In the case of a finite noise intensity one can easily see that [15]

$$
\hat{C}(s) \sim K+o(1) \quad(s \rightarrow 0),
$$

where $K$ is given by Eq. (3.1).

(b) Long-time tail noise. Now the correlation function decays as a power law of the form

$$
C(\tau) \sim \frac{A}{\tau^{\alpha}} \quad(\tau \rightarrow \infty),
$$

where $0<\alpha<1$. The Laplace transform of the correlation function behaves as [15]

$$
\hat{C}(s) \sim \frac{A \Gamma(1-\alpha)}{s^{1-\alpha}} \quad(s \rightarrow 0) .
$$

As we have mentioned in Sec. II we will only consider dissipative kernels $\beta(t)$ that lead to stationary states for the velocity process. Moreover, we restrict ourselves to those kernels $\beta(t)$ leading to relaxation functions, $h(t)$, that finally become monotonic, that is, there exists some $t_{0} \geqslant 0$ such that $d h(t) / d t$ has definite sign for all $t \geqslant t_{0}$. Again, 
$\beta(t)$ is supposed to be locally integrable. Equation (2.6) suggests considering the following cases:

(a) Finite relaxation time. That is, the inverse of the relaxation time

$$
\int_{0}^{\infty} \beta(t) d t=E<\infty
$$

is finite and positive. In this case [see Eq. (3.2)]

$$
\hat{\beta}(s) \sim E+o(1) \quad(s \rightarrow 0) .
$$

(b) Slow relaxation. We now assume that the friction kernel decays as

$$
\beta(\tau) \sim \frac{B}{\tau^{\gamma}} \quad(\tau \rightarrow \infty)
$$

where $0<\gamma<1$. Therefore, its Laplace transform behaves as [see Eq. (3.4)]

$$
\hat{\beta}(s) \sim \frac{B \Gamma(1-\gamma)}{s^{1-\gamma}} \quad(s \rightarrow 0) .
$$

The behavior of $\hat{h}(s)$ is readily obtained from Eq. (2.6) and from the expansions of $\hat{\beta}(s)$ when $s \rightarrow 0$ [cf. Eqs. (3.6) and (3.8)]. We now want to know the long-time behavior of $h(t)$ and $H(t)$. This is accomplished by the use of Tauberian theorems, which can be considered converse Abelian theorems and they consist of a collection of results that roughly give the asymptotic behavior of a function $f(t)$ as $t \rightarrow \infty$ if one knows the small $s$ behavior of its Laplace transform [14]. For the two kinds of memory friction introduced above we have [16] the following:

(a) Finite relaxation time. In this case,

$$
\hat{h}(s) \sim \frac{1}{E}, \quad \hat{H}(s) \sim \frac{1}{E s} \quad(s \rightarrow 0)
$$

and

$$
h(t)=o\left(t^{-1}\right), \quad H(t) \sim \frac{1}{E} \quad(t \rightarrow \infty) .
$$

(b) Slow relaxation. Now we have

$$
\hat{h}(s) \sim \frac{s^{1-\gamma}}{B \Gamma(1-\gamma)}, \quad \hat{H}(s) \sim \frac{s^{-\gamma}}{B \Gamma(1-\gamma)} \quad(s \rightarrow 0)
$$

and

$$
\begin{gathered}
h(t) \sim-\frac{(1-\gamma) \sin (\gamma \pi)}{B \pi} t^{\gamma-2}, \\
H(t) \sim \frac{\sin (\gamma \pi)}{B \pi} t^{\gamma-1} \quad(t \rightarrow \infty) .
\end{gathered}
$$

In Appendix B we verify these relations for a particular example.

The substitution of expansions (3.10) and (3.12) into Eqs. (2.8) and (2.9) allows us to obtain the long-time behavior of the variances of process (1.1). We can easily see that the variance of the velocity becomes a constant for all cases discussed above. Moreover, the average velocity converge to zero because $h(t)$ goes to zero as $t \rightarrow \infty$. Hence, the velocity process reaches a stationary state with zero average value. When the driving noise is internal then the stationary state is the equilibrium state. In this case the variance of the velocity can be obtained in a more straightforward way. This is the case, for instance, of a free Brownian particle of unit mass in a medium at temperature $T$ where

$$
\lim _{t \rightarrow \infty} \sigma_{v v}^{2}(t)=k_{B} T
$$

The behavior of the mean square displacement, $\sigma_{x x}^{2}(t)$, shows a much richer diversity. The normal diffusion behavior arises when both the intensity of the noise $K$, Eq. (3.1), and the inverse relaxation time $E$, Eq. (3.5), are finite. In such a case one obtains from Eq. (2.8) that

$$
\sigma_{x x}^{2}(t) \sim \frac{2 K}{E^{2}} t \quad(t \rightarrow \infty) .
$$

Let us now derive this expression. We first note that Eq. (2.8) can be written as

$$
\sigma_{x x}^{2}(t)=2 t^{2} \int_{0}^{1} H(t z) d z \int_{0}^{z} H\left(t z^{\prime}\right) C\left(t z-t z^{\prime}\right) d z^{\prime} .
$$

From Eq. (3.10), we see that, for friction kernels of type (a), $H(t)$ converges to $1 / E$ when $t \rightarrow \infty$. Therefore,

$$
\sigma_{x x}^{2}(t) \sim \frac{2 t^{2}}{E^{2}} \int_{0}^{1} d z \int_{0}^{z} C\left(t z-t z^{\prime}\right) d z^{\prime} .
$$

Reversing the order of integration we get

$$
\sigma_{x x}^{2}(t) \sim \frac{2 t}{E^{2}} \int_{0}^{t} C(x)\left(1-\frac{x}{t}\right) d x .
$$

When $t \rightarrow \infty$ we finally have

$$
\sigma_{x x}^{2}(t) \sim \frac{2 t}{E^{2}} \int_{0}^{\infty} C(x) d x
$$

and for driving noises with finite intensity $K$ we obtain Eq. (3.14).

When the correlation function presents a long-time tail and $E$ is finite, the $X(t)$ process is superdiffusive because

$$
\sigma_{x x}^{2}(t) \sim \frac{2 A}{E^{2}(1-\alpha)(2-\alpha)} t^{2-\alpha} \quad(t \rightarrow \infty)
$$

and $0<\alpha<1$. The derivation of this expression is outlined in Appendix C. The existence of the tail indicates that the random force varies slowly and persists driving the system in the same direction. As a consequence, the system becomes superdiffusive.

Nevertheless, when the relaxation is also slow [i.e., $\beta$ of type (b)] then the number of possible limiting behaviors increases notably. Thus when the driving noise has a finite 
intensity $K$ then the long-time behavior of $X(t)$ depends solely on the exponent $\gamma$ of the relaxation tail. The possible limiting behaviors of the variance, as a function $\gamma$, are (cf. Appendix $\mathrm{C}$ )

$$
\sigma_{x x}^{2}(t) \sim\left\{\begin{array}{lll}
\text { const, } & 0<\gamma<1 / 2 & \text { (stationary) } \\
\frac{2 K \sin ^{2}(\gamma \pi)}{B^{2} \pi^{2}} \ln t, & \gamma=1 / 2 & \text { (logarithmic) } \\
\frac{2 K \sin ^{2}(\gamma \pi)}{B^{2} \pi^{2}(2 \gamma-1)} t^{2 \gamma-1} & 1 / 2<\gamma<1 & \text { (subdiffusion) }
\end{array}\right.
$$

$(t \rightarrow \infty)$. It is remarkable that for $\gamma=1 / 2$ the mean square displacement grows as slowly as the logarithmic behavior.

When both the correlation function and the memory kernel show long-time tails the process may become asymptotically stationary to superdiffusive, including subdiffusive, diffusive, and logarithmic. The fact that no fluctuation-dissipation relation holds means that the exponents $\alpha$ and $\gamma$ are independent. Thus the long-time behavior of the variance $\sigma_{x x}^{2}(t)$ will depend on both exponents. Following a similar reasoning we have (see Appendix C)

$$
\sigma_{x x}^{2}(t) \sim\left\{\begin{array}{lll}
\text { const, } & 2 \gamma<\alpha & \text { (stationary) } \\
\frac{2 A \sin (\gamma \pi) \Gamma(1-2 \gamma)}{B^{2} \pi \Gamma^{2}(1-\gamma)} \ln t, & 2 \gamma=\alpha & \text { (logarithmic) } \\
\frac{2 A \sin (\gamma \pi) \Gamma(1-\alpha)}{B^{2} \pi \Gamma(1-\gamma) \Gamma(1-\alpha+\gamma)(2 \gamma-\alpha)} t^{2 \gamma-\alpha} & 2 \gamma>\alpha & \text { (anomalous diffusion) }
\end{array}\right.
$$

$(t \rightarrow \infty)$. In the third case where $2 \gamma>\alpha$ we distinguish three different regimes: (i) subdiffusion, if $2 \gamma-\alpha<1$; (ii) normal diffusion, if $2 \gamma-\alpha=1$; and (iii) superdiffusion, if $2 \gamma-\alpha>1$. We show in Fig. 1 the long-time behavior of the mean square displacement as a function of the exponents $\alpha$ and $\gamma$.

We note that for internal noise, the fluctuation-dissipation relation implies that the tail exponents of $C(t)$ and $\beta(t)$ are equal, that is, $\alpha=\gamma$. In this case, the long-time behavior of

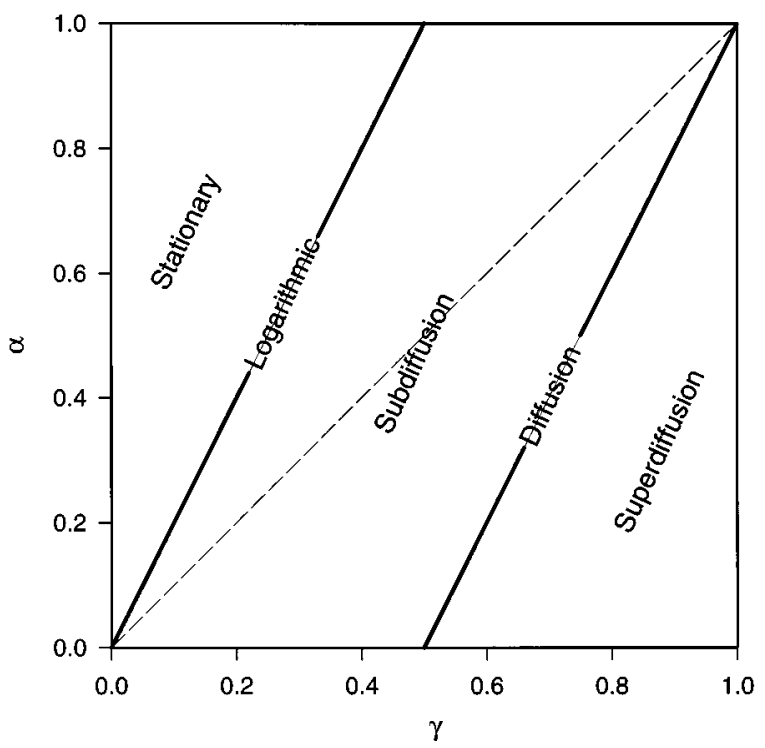

FIG. 1. Asymptotic behavior of $\sigma_{x x}^{2}(t)$ as a function of exponents $\alpha$ and $\gamma$ for $C(\tau) \sim \tau^{-\alpha}$ and $\beta(\tau) \sim \tau^{-\gamma}$ when $\tau \rightarrow \infty$. Logarithmic behavior arises for $\gamma=2 \alpha$ and the diffusive one for $\gamma=2 \alpha-1$. The discontinuous line corresponds to the internal noise case (see text).
$X(t)$ is always subdiffusive with

$$
\sigma_{x x}^{2}(t) \sim \frac{2\left(k_{B} T\right)^{2} \sin (\gamma \pi)}{A \gamma \pi} t^{\gamma}
$$

This result can also be obtained in a different way [9]. We first observe that the properties of the mean square displacement are determined by the velocity process. In effect, the variance of the displacement can be written in the form

$$
\sigma_{x x}^{2}(t)=2 \int_{0}^{t} d t^{\prime} \int_{0}^{t^{\prime}} C_{v}\left(t^{\prime}, t^{\prime \prime}\right) d t^{\prime \prime}
$$

where $C_{v}\left(t^{\prime}, t^{\prime \prime}\right) \equiv\left\langle\dot{X}(t) \dot{X}\left(t^{\prime}\right)\right\rangle$ is the velocity autocorrelation function. When $t \rightarrow \infty$, the velocity becomes a stationary random process and $C_{v}\left(t^{\prime}, t^{\prime \prime}\right)$ will be a function of $t^{\prime}-t^{\prime \prime}$. Hence from Eq. (3.23) we get

$$
\sigma_{x x}^{2}(t) \sim 2 \int_{0}^{t} C_{v}(\tau)(t-\tau) d \tau \quad(t \rightarrow \infty) .
$$

For the case of internal noise we have shown in Appendix A that $C_{v}(\tau)=k_{B} T h(t)$, [cf. Eq. (1.5)]. Therefore,

$$
\sigma_{x x}^{2}(t) \sim 2 k_{B} T \int_{0}^{t} h(\tau)(t-\tau) d \tau \quad(t \rightarrow \infty) .
$$

We define

$$
\begin{gathered}
I_{1}(t) \equiv 2 k_{B} T t \int_{0}^{t} h(\tau) d \tau, \\
I_{2}(t) \equiv-2 k_{B} T \int_{0}^{t} h(\tau) \tau d \tau .
\end{gathered}
$$


Taking into account Eq. (3.12) we see that the long-time behavior of $I_{2}(t)$ is

$$
I_{2}(t) \sim 2 k_{B} T \frac{(1-\gamma) \sin (\gamma \pi)}{B \gamma \pi} t^{\gamma} \quad(t \rightarrow \infty) .
$$

The asymptotic behavior of $I_{1}(t)$ is obtained as follows. We first write $I_{1}(t)$ in the form

$$
I_{1}(t)=2 k_{B} T t\left[\int_{0}^{\infty} h(\tau) d \tau-\int_{t}^{\infty} h(\tau) d \tau\right] .
$$

The asymptotic expression of the first integral on the righthand side of this equation is obtained by taking the limit when $s \rightarrow 0$ of $\hat{h}(s)$ [17]. From Eq. (3.11), we see that

$$
\int_{0}^{\infty} h(t) d t=\lim _{s \rightarrow 0} \hat{h}(s)=0 .
$$

Using Eq. (3.12) we see that as $t \rightarrow \infty$ the second integral in Eq. (3.29) behaves as

$$
\int_{t}^{\infty} h(\tau) d \tau \sim-\frac{\sin (\gamma \pi)}{B \pi} t^{\gamma-1} \quad(t \rightarrow \infty) .
$$

Hence,

$$
\sigma_{x x}^{2}(t) \sim I_{1}(t)+I_{2}(t) \sim 2 k_{B} T \frac{\sin (\gamma \pi)}{B \gamma \pi} t^{\gamma} \quad(t \rightarrow \infty) .
$$

Taking into account that the quantities $A$ and $B$ are related by $A=k_{B} T B$ we finally obtain Eq. (3.22).

In the next section we work out exactly two cases where the long-time behavior becomes diffusive.

\section{EXAMPLES}

We discuss in this section two examples that can be solved analytically. The expression for the mean square displacement at all times makes it possible to identify different regimes in the behavior of these systems. Unfortunately, we have not been able to solve exactly any case with a long-time tail. In a previous study, Muralidhar et al. reduced the velocity correlation function for a power law tail kernel up to quadrature [18]. This particular case refers to internal noise and the quadrature has to be approximated in order to obtain the standard anomalous diffusive behavior.

We now assume that the stationary driving noise $F(t)$ is exponentially correlated,

$$
C\left(t-t^{\prime}\right)=\frac{D}{2 \tau_{c}} e^{-\left|t-t^{\prime}\right| / \tau_{c}},
$$

where $D$ is the noise intensity and $\tau_{c}$ is the correlation time. This is a relevant example of driving noise with finite intensity.

\section{A. Internal noise}

Let us first assume that $F(t)$ is internal noise. In this case the frictional kernel is given by $\beta(t)=C(t) / k_{B} T$ (note that this example represents a Brownian particle). The function $H(t)$ is given by [cf. Eq. (2.3)]

$$
H(t)=\frac{2 k_{B} T}{D}\left[1-A e^{-t / 2 \tau_{c}} \sin (\lambda t+\alpha)\right],
$$

where

$$
\lambda=\sqrt{\frac{D}{2 k_{B} T \tau_{c}}-\frac{1}{4 \tau_{c}^{2}}}, \quad \alpha=\arctan \left(\frac{2 \lambda \tau_{c}}{1-D \tau_{c} / k_{B} T}\right),
$$

and

$$
A=\lambda+\frac{1}{4 \lambda \tau_{c}^{2}}\left(1-\frac{D \tau_{c}}{k_{B} T}\right)^{2} .
$$

The substitution of Eq. (4.2) into Eqs. (2.11)-(2.13) results, after some algebra, in the following explicit expressions for the variances of the particle

$$
\begin{gathered}
\sigma_{v v}^{2}(t)=k_{B} T-\frac{\left(k_{B} T\right)^{3}}{D^{2}} A^{2} e^{-t / \tau_{c}}\left[4 \lambda^{2}+\left(\frac{1}{\tau_{c}^{2}}-4 \lambda^{2}\right) \sin ^{2}(\lambda t+\alpha)-\frac{2 \lambda}{\tau_{c}} \sin 2(\lambda t+\alpha)\right], \\
\sigma_{x x}^{2}(t)=\frac{4\left(k_{B} T\right)^{2}}{D} t-\frac{4\left(k_{B} T\right)^{3}}{D^{2}}\left[1+A\left(\sin \alpha+2 \lambda \tau_{c} \cos \alpha\right)\right]+\frac{4\left(k_{B} T\right)^{3}}{D^{2}} A e^{-t / 2 \tau_{c}\left[3 \sin (\lambda t+\alpha)+2 \lambda \tau_{c} \cos (\lambda t+\alpha)\right.} \\
\left.-A e^{-t / 2 \tau_{c}} \sin ^{2}(\lambda t+\alpha)\right],
\end{gathered}
$$

and

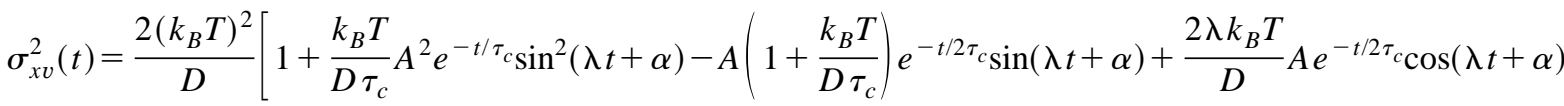

$$
\begin{aligned}
& -\frac{\lambda k_{B} T}{D} A^{2} e^{\left.-t / \tau_{c} \sin 2(\lambda t+\alpha)\right] .}
\end{aligned}
$$


We see that when $t \rightarrow \infty$,

$$
\sigma_{v v}^{2}(t) \sim k_{B} T, \quad \sigma_{x x}^{2}(t) \sim\left[4\left(k_{B} T\right)^{2} / D\right] t .
$$

This result is in agreement with Eqs. (3.13) and (3.14) because now $K=D / 2$ and $E=D / 2 k_{B} T$.

Since, in this case, we know exactly the time evolution of the variances for all times, we can identify several regimes. We first distinguish three different time scales involved in the motion of the Brownian particle. Namely, the observation time $t$, the relaxation time $\tau_{r}$ (the inverse of $E$ ), and the correlation time of the input noise [which is related with the correlation function $C(t)]$. Note that for internal driving noise the correlation time and the relaxation time are essentially of the same order of magnitude [cf. Eq. (1.3)], that is, $\tau_{r} \sim \tau_{c} \equiv \tau$. Hence, the behavior of the variances will depend on whether $t$ and $\tau$ are similar or not comparable. In this case we have only two extreme situations (i) $t \ll \tau$, and (ii) $t \gg \tau$. Case (i) obviously corresponds to $t \rightarrow 0$ while case (ii) is equivalent to $t \rightarrow \infty$. From Eqs. (4.5) and (4.6), we see that

$$
\sigma_{x x}^{2}(t) \sim\left(D / 8 \tau_{c}\right) t^{4}, \quad \sigma_{v v}^{2}(t) \sim\left(D / 2 \tau_{c}\right) t^{2} \quad(t \ll \tau) .
$$

and

$$
\sigma_{x x}^{2}(t) \sim \frac{4\left(k_{B} T\right)^{2}}{D} t, \quad \sigma_{v v}^{2}(t) \sim k_{B} T \quad(t \gg \tau) .
$$

Let $\nu_{x}$ and $\nu_{v}$ be exponents defined by $\sigma_{x x}^{2}(t) \sim t^{\nu_{x}}$ and $\sigma_{v v}^{2}(t) \sim t^{\nu_{v}}$. We may then summarize the above results with the following transitions between exponents (as $t$ increases)

$$
\nu_{x}=4 \rightarrow \nu_{x}=1, \quad \nu_{v}=2 \rightarrow \nu_{v}=0
$$

\section{B. Markovian relaxation}

Suppose now that the driving noise is external. In this case $C(t)$ and $\beta(t)$ are not related. Let us further assume that the relaxation is Markovian, that is, $\beta(t)=\beta \delta(t)$. In this case,

$$
h(t)=e^{-\beta t}, \quad H(t)=\left(1-e^{-\beta t}\right) / \beta
$$

The substitution of Eq. (4.12) and Eq. (4.1) into Eqs. (2.8)(2.10) yields

$$
\begin{aligned}
& \sigma_{v v}^{2}(t)=\frac{D}{2 \beta}\left\{\frac{1}{1+\beta \tau_{c}}+\frac{2 \beta \tau_{c}}{1-\beta^{2} \tau_{c}^{2}} e^{-\left(\beta+1 / \tau_{c}\right) t}-\frac{1}{1-\beta \tau_{c}} e^{-2 \beta t}\right\} \\
& \sigma_{x x}^{2}(t)=\frac{D}{\beta^{2}}\left\{t-\frac{\beta \tau_{c}^{2}}{1-\beta \tau_{c}} e^{-t / \tau_{c}}+\frac{\beta \tau_{c}^{2}}{1-\beta^{2} \tau_{c}^{2}} e^{-\left(\beta+1 / \tau_{c}\right) t}+\frac{2-\beta \tau_{c}}{\beta\left(1-\beta \tau_{c}\right)} e^{-\beta t}-\frac{1}{2 \beta\left(1-\beta \tau_{c}\right)} e^{-2 \beta t}-\frac{2 \beta^{2} \tau_{c}^{2}+4 \beta \tau_{c}+3}{2 \beta\left(1+\beta \tau_{c}\right)}\right\},
\end{aligned}
$$

and

$$
\sigma_{x v}^{2}(t)=\frac{D}{2 \beta}\left\{\frac{1}{\beta}+\frac{\tau_{c}}{1-\beta \tau_{c}} e^{-t / \tau_{c}}-\frac{\tau_{c}}{1-\beta \tau_{c}} e^{-\left(\beta+1 / \tau_{c}\right) t}-\frac{2-\beta \tau_{c}}{\beta\left(1-\beta \tau_{c}\right)} e^{-\beta t}+\frac{1}{\beta\left(1-\beta \tau_{c}\right)} e^{-2 \beta t}\right\}
$$

Let us analyze the asymptotic properties of the variances. We first observe that in this case there are three different time scales: the correlation time $\tau_{c}$, the relaxation time $\tau_{r}=\beta^{-1}$, and the observation time $t$. We will now show that the behavior of the variances depends on whether these three time scales are similar or not comparable. For $t$ smaller than any other time scale, it follows from Eqs. (4.13) and (4.14) that

$$
\sigma_{x x}^{2}(t) \sim \frac{D}{8 \tau_{c}} t^{4}, \quad \sigma_{v v}^{2}(t) \sim \frac{D}{2 \tau_{c}} t^{2} \quad(t \rightarrow 0) .
$$

On the other hand, if $t$ is larger than any time scale, then

$$
\sigma_{x x}^{2}(t) \sim \frac{D}{\beta^{2}} t, \quad \sigma_{v v}^{2}(t) \sim \frac{D}{2 \beta\left(1+\beta \tau_{c}\right)} \quad(t \rightarrow \infty) .
$$

Let us now investigate the intermediate-time behavior of the variances between these two extreme situations. We first assume that $\tau_{c}$ and $\tau_{r}$ are not comparable. In this situation we have two different cases.
(1) $\tau_{c} \ll \tau_{r}$. In this case we distinguish the time regime where the observation time is much longer than the correlation time but still smaller than the relaxation time: $\tau_{c} \ll t<\tau_{r}$. In this regime $F(t)$ acts as white noise, with $C(t)=D \delta(t)$, and from Eqs. (2.9)-(4.12) we get (recall that $\beta t<1)$

$$
\sigma_{x x}^{2}(t) \sim \frac{1}{3} D t^{3}, \quad \sigma_{v v}^{2}(t) \sim D t
$$

Therefore, when $\tau_{c} \ll \beta^{-1}$, we have the following transition (as $t$ increases)

$$
\nu_{x}=4 \rightarrow \nu_{x}=3 \rightarrow \nu_{x}=1, \quad \nu_{v}=2 \rightarrow \nu_{v}=1 \rightarrow \nu_{v}=0 .
$$

(2) $\tau_{c} \gg \tau_{r}$. In this case we distinguish the time regime where the observation time is much longer than the relaxation time but smaller than the correlation time: $\tau_{r} \ll t<\tau_{c}$. In this regime the inertial term may be neglected and the 
dynamical equation can be approximated by $\dot{X} \approx \beta^{-1} F(t)$. As a consequence the variances are given by (recall that $\left.t / \tau_{c}<1\right)$

$$
\sigma_{x x}^{2}(t) \sim \frac{D}{2 \beta^{2} \tau_{c}} t^{2}, \quad \sigma_{v v}^{2}(t) \sim \frac{D}{2 \beta^{2} \tau_{c}},
$$

and the transitions between exponents are given by

$$
\nu_{x}=4 \rightarrow \nu_{x}=2 \rightarrow \nu_{x}=1, \quad \nu_{v}=2 \rightarrow \nu_{v}=0,
$$

where now $\tau_{c} \gg \beta^{-1}$.

We also observe that when the time scales are similar, $\tau_{c} \sim \tau_{r}$ (as is the case of internal driving noise), we are only able to distinguish two extreme asymptotic regimes: $t \rightarrow 0$ and $t \rightarrow \infty$. This results in the transition given by Eq. (4.11).

\section{PROBABILITY DENSITY FUNCTIONS}

We now assume that the input noise is Gaussian. Due to linearity of Eq. (1.1), we see that the joint probability density function $p(x, v, t)$ for a displacement $x$ and a velocity $v$ at time $t$ must be Gaussian. As a consequence, the joint characteristic function of the process can be written as

$$
\begin{aligned}
\tilde{p}(\mu, \rho, t)= & \exp \left\{i\langle X(t)\rangle \mu+i\langle\dot{X}(t)\rangle \rho-\frac{1}{2}\left[\sigma_{x x}^{2}(t) \mu^{2}\right.\right. \\
& \left.\left.+2 \sigma_{x v}^{2}(t) \mu \rho+\sigma_{v v}^{2}(t) \rho^{2}\right]\right\} .
\end{aligned}
$$

Using the method of the characteristic function [8] or the method of functional derivative [6], one can easily see that the joint probability density function $p(x, v, t)$ obeys the following Fokker-Planck equation:

$$
\begin{aligned}
\left(\frac{\partial}{\partial t}+v \frac{\partial}{\partial x}\right) p(x, v, t)= & \xi(t) \frac{\partial}{\partial v} v p(x, v, t) \\
& +\varphi(t) \frac{\partial^{2}}{\partial v^{2}} p(x, v, t) \\
& +\psi(t) \frac{\partial^{2}}{\partial x \partial v} p(x, v, t),
\end{aligned}
$$

where

$$
\begin{gathered}
\xi(t)=-\dot{h}(t) / h(t), \\
\varphi(t)=\xi(t)+\dot{\sigma}_{v v}^{2}(t) / 2,
\end{gathered}
$$

and

$$
\psi(t)=-\sigma_{v v}^{2}(t)+\xi(t) \sigma_{x v}^{2}(t)+\dot{\sigma}_{v v}^{2}(t) .
$$

In the case of internal driving noise, these expressions reduce to [12]

$$
\begin{gathered}
\xi(t)=-\dot{h}(t) / h(t), \\
\varphi(t)=k_{B} T \xi(t),
\end{gathered}
$$

and

$$
\psi(t)=k_{B} T[h(t)-1+\xi(t) H(t)] .
$$

Let us now obtain the marginal probability density functions of the velocity $p(v, t)$ and the displacement $p(x, t)$. Setting $\mu=0$ in Eq. (5.1) and following the method of the characteristic function, we can see that $p(v, t)$ obeys the following Fokker-Planck equation:

$$
\frac{\partial p(v, t)}{\partial t}=-\xi(t) \frac{\partial}{\partial v} v p(v, t)+\frac{1}{2} D_{v}(t) \frac{\partial^{2} p(v, t)}{\partial v^{2}},
$$

where

$$
D_{v}(t)=\varphi(t) .
$$

The marginal density of the displacement $p(x, t)$ obeys the Fokker-Planck equation

$$
\frac{\partial p(x, t)}{\partial t}=-\langle v(t)\rangle \frac{\partial p(x, t)}{\partial x}+\frac{1}{2} D_{x}(t) \frac{\partial^{2} p(x, t)}{\partial x^{2}},
$$

where $D_{x}(t)$ is the time derivative of the variance, i.e.,

$$
D_{x}(t)=\dot{\sigma}_{x x}^{2}(t)=2 \sigma_{x v}^{2}(t) .
$$

\section{CONCLUSIONS}

We have analyzed the long-time behavior of processes obeying a generalized Langevin equation. When the driving noise is external several behaviors arise depending on the memory kernel and the correlation function. The common diffusive behavior appears when both the relaxation time and the correlation are finite and nonvanishing.

In the presence of long time tails, either in the memory kernel or in the correlation function, the system diffuses anomalously. The exact nature of the leading behavior depends on the exponents of the long-time tails of both the friction kernel and the correlation function. Thus, the possible behaviors include stationary state, logarithmic growth, subdiffusion, normal diffusion, and superdiffusion. Figure 1 summarizes the limiting states as a function of the exponents of the long-time tails. However, we have shown that when the driving noise is internal the final state is always subdiffusive.

At this point we mention that the problem of anomalous diffusion has been studied mainly for particles moving on fractals and other disordered media. In this context subdiffusion appears due to trapping sites where particles disappear, or to excursions of the particles along blobs or dead ends. Most of the results in this area are known by a combination of scaling arguments and simulation results $[1,2]$. However, superdiffusion seems to be more difficult to explain although models based on Levy flights have successfully explained it for some systems [19]. A superdiffusive behavior with exponent $\nu_{x}=3$ has been proved nonetheless for free inertial particles driven by Gaussian white noise [4]. In any case it is quite difficult to obtain general anomalous diffusion behaviors with exponents $\nu_{x}>1$. On the other hand, subdiffusion appears for particles moving on a percolation cluster for which the dynamics can be described by a generalized 
Langevin equation with a slow relaxation kernel $[9,18]$. In such a case the noise is internal and we have proved that the behavior of the system is subdiffusive. Therefore, our main objective in this paper has been to extend this last result to external driving noise where the system not only shows subdiffusion but all kinds of diffusive behavior (e.g., logarithmic, subdiffusion, normal diffusion, and superdiffusion).

Finally, when the noise is Gaussian, the expressions for the joint and marginal probability densities can be derived. These densities satisfy the Fokker-Planck equation with time dependent coefficients. The time dependence is the signature of the non-Markovian character of the process. As an example, we have exactly solved two cases and study the behavior of the mean square displacement at all times. When the time scales of the process are completely separated, there is a new transition from the ballistic behavior to the final diffusive behavior. This intermediate state is characterized by an exponent $\nu_{x}$ that takes the value 2 or 3 depending on the fundamental time scales $\tau_{r}$ and $\tau_{c}$.

\section{ACKNOWLEDGMENTS}

This work was supported in part by Dirección General de Investigación Científica y Técnica under Contract No. PB930812, by Societat Catalana de Física (Institut d'Estudis Catalans). K.G.W. acknowledges the financial support of the Ministerio de Educación y Ciencia of Spain.

\section{APPENDIX A: VELOCITY CORRELATION FUNCTION FOR INTERNAL NOISE}

We assume that the velocity $\dot{X}(t)$ relaxes to a stationary state. The correlation function of this process will be

$$
C_{v}(\tau)=\lim _{t \rightarrow \infty}\langle\dot{X}(t+\tau) \dot{X}(t)\rangle
$$

From Eq. (2.5) and by taking into account the correlation function $C(\tau)$ of the driving noise, we write

$$
C_{v}(\tau)=\int_{0}^{\infty} h\left(t^{\prime}\right) d t^{\prime} \int_{0}^{\infty} h\left(t^{\prime \prime}\right) C\left(\tau-t^{\prime}+t^{\prime \prime}\right) d t^{\prime \prime}
$$

The Fourier transform of $C_{v}(\tau)$ reads

$$
\hat{C}_{v}(\omega)=|\hat{h}(i \omega)|^{2} \hat{C}(\omega)
$$

where $\hat{h}(i \omega)$ is the Laplace transform of $h(t)$ evaluated at $s=i \omega$. When the driving noise is internal, $C(\tau)=k_{B} T \beta(\tau)$ and

$$
\hat{C}(\omega)=2 k_{B} T \operatorname{Re}[\hat{\beta}(i \omega)]
$$

where $\operatorname{Re}(z)$ is the real part of $z$ and $\hat{\beta}(s)$ is the Laplace transform of $\beta(t)$. Substituting Eq. (2.6) into Eq. (A4) and using the symmetry property of $C(\tau)$ yield

$$
C_{v}(\tau)=k_{B} T h(t)
$$

On the other hand, we know that $\left\langle\dot{X}^{2}(t)\right\rangle \rightarrow k_{B} T$ as $t \rightarrow \infty$. Whence

$$
h(\tau)=\lim _{t \rightarrow \infty} \frac{\langle\dot{X}(t+\tau) \dot{X}(t)\rangle}{\langle\dot{X}(t) \dot{X}(t)\rangle} .
$$

\section{APPENDIX B: RELAXATION FUNCTIONS FOR A LONG-TIME TAIL KERNEL}

As an example, the asymptotic expansions of functions $h(t)$ and $H(t)$, Eq. (3.12), can be checked for the following relaxation kernel:

$$
\beta(t)=1 / \sqrt{\pi t}
$$

The Laplace transform of this reads

$$
\hat{\beta}=1 / \sqrt{s}
$$

and from Eqs. (2.3) and (2.6) we have

$$
\hat{H}(s)=\frac{1}{s^{2}+s^{1 / 2}}, \quad \hat{h}(s)=\frac{s^{1 / 2}}{s^{3 / 2}+1} .
$$

The Laplace inversion yields

$$
\begin{aligned}
H(t)= & \frac{4}{3}\left[\frac{\sqrt{3}}{2} \sin (\sqrt{3} t / 2)-\frac{1}{2} \cos (\sqrt{3} t / 2)\right] e^{-t / 2} \\
& +\frac{1}{\pi} \int_{0}^{\infty} e^{-r t} \frac{r^{-1 / 2}}{1+r^{3}} d r,
\end{aligned}
$$

and

$$
h(t)=\frac{4}{3} \cos (\sqrt{3} t / 2) e^{-t / 2}-\frac{1}{\pi} \int_{0}^{\infty} e^{-r t} \frac{r^{1 / 2}}{1+r^{3}} d r
$$

which agrees with previous results [18]. The long-time behavior can be obtained from these expressions. In effect, when $t \rightarrow \infty$, the first term of the expression for $h(t)$ decays exponentially while the maximum contribution of the second term comes from $r$ around zero. Therefore,

$$
h(t) \sim-\frac{1}{\pi} \int_{0}^{\infty} e^{-r t} r^{1 / 2} d r=-\frac{1}{2 \sqrt{\pi}} t^{-3 / 2},
$$

as $t \rightarrow \infty$. Likewise, it can be demonstrated from Eq. (B4) that

$$
H(t) \sim \frac{1}{\sqrt{\pi}} t^{-1 / 2}
$$

We can now test that the latter results are in agreement with the general expansion Eq. (3.12). The kernel of this example is of type (b) (see Sec. III) with $\gamma=1 / 2$ and $B=1 / \sqrt{\pi}$. Then, Eq. (3.12) for these values results in expansions (B6) and (B7). 


\section{APPENDIX C: ASYMPTOTIC BEHAVIOR OF THE VARIANCE}

We start from Eq. (2.8) and write

$$
\sigma_{x x}^{2}(t)=2 \int_{0}^{t} H\left(t^{\prime}\right) \rho\left(t^{\prime}\right) d t^{\prime},
$$

where

$$
\rho(t)=\int_{0}^{t} H\left(t^{\prime}\right) C\left(t-t^{\prime}\right) d t^{\prime} .
$$

The Laplace transform of this equation reads

$$
\hat{\rho}(s)=\hat{H}(s) \hat{C}(s) .
$$

The long-time behavior of $\rho(t)$ can be obtained from its Laplace transform because it is the convolution of two functions, $H(t)$ and $C(t)$, both having an asymptotic expansion [16].

Let us now prove Eq. (3.19). Assume that $\beta(t)$ is of type (a) ( $E$ is finite) and that $C(\tau)$ shows a long tail [cf. Eq. (3.3)]. Then,

$$
\hat{\rho}(s)=\hat{H}(s) \hat{C}(s) \sim \frac{1}{E s} \frac{A \Gamma(1-\alpha)}{s^{1-\alpha}} \quad(s \rightarrow 0) .
$$

Using a Tauberian theorem [16] we have

$$
\rho(t) \sim \frac{A \Gamma(1-\alpha)}{E \Gamma(2-\alpha)} t^{1-\alpha} \quad(t \rightarrow \infty) .
$$

After substituting this equation into Eq. (C1), we get

$$
\sigma_{x x}^{2}(t) \sim \frac{2 A}{E^{2}(1-\alpha)(2-\alpha)} t^{2-\alpha} \quad(t \rightarrow \infty),
$$

which agrees with Eq. (3.19).

When the friction kernel is of type (b), there are more possibilities. Let us discuss the case of a driving noise of finite intensity, $K$, and a power law kernel of exponent $\gamma$ [cf. Eq. (3.7)]. The Laplace transform of function $\rho(t)$ behaves for small $s$ as

$$
\hat{\rho}(s) \sim K \frac{s^{-\gamma}}{B \Gamma(1-\gamma)} .
$$

Let us obtain the long-time behavior of $\rho(t)$. From our assumptions we know that $h(t)$, and consequently $H(t)$, becomes monotonic when $t \geqslant t_{0}$ (for some $t_{0} \geqslant 0$ ). As a consequence $H(t)$ possesses an asymptotic expansion (as $t$ goes to infinity) that allows us to apply a convenient Tauberian theorem to the expression for $\hat{\rho}(s)$ given by Eq. (C7). Therefore, the long-time behavior of $\rho(t)$ reads [16]

$$
\rho(t) \sim K \frac{\sin (\gamma \pi)}{B \pi} t^{\gamma-1}, \quad t \rightarrow \infty .
$$

Once this expansion is substituted into Eq. (3.1) along with the asymptotic expansion of $H(t)$ [cf. Eq. (3.12)] the leading behavior of $\sigma_{x x}^{2}(t)$ is given by the integral

$$
\sigma_{x x}^{2}(t) \sim \frac{2 K \sin ^{2}(\gamma \pi)}{B^{2} \pi^{2}} \int^{t} t^{\prime 2 \gamma-2} d t^{\prime},
$$

where the lower limit of integration is irrelevant. The reason for different limiting behaviors of the mean square displacement now becomes clear. In effect, the integral above grows as $t \rightarrow \infty$ in three different ways. Thus, if $2 \gamma-2<-1$, the integral converges to a finite value. In this case, $\sigma_{x x}^{2}(t)$ reaches a constant value and the process $X(t)$ becomes stationary. On the other hand, when $2 \gamma-2>-1$, the integral diverges as $t^{2 \gamma-1}$, and

$$
\sigma_{x x}^{2}(t) \sim \frac{2 K \sin ^{2}(\gamma \pi)}{B^{2} \pi^{2}(2 \gamma-1)} t^{2 \gamma-1} \quad(t \rightarrow \infty) .
$$

An intermediate case arises when $2 \gamma-2=-1$, that is if $\gamma=1 / 2$. Now, the integrant in Eq. (C9) decays as $t^{-1}$ for large $t$ and the integral diverges as a logarithm. Hence

$$
\sigma_{x x}^{2}(t) \sim \frac{2 K \sin ^{2}(\gamma \pi)}{B^{2} \pi^{2}} \ln t \quad(t \rightarrow \infty) .
$$

The derivation when both the friction kernel and the correlation function present a long-time tail is obtained in a similar way.
[1] S. Havlin and D. Ben-Avraham, Adv. Phys. 36, 695 (1987).

[2] J. P. Bouchaud and A. Georges, Phys. Rep. 195, 127 (1990).

[3] T. Bohr and A. Pikovsky, Phys. Rev. Lett. 70, 2892 (1993).

[4] J. Masoliver, Phys. Rev. A 45, 706 (1992).

[5] J. Masoliver, Phys. Rev. E 48, 121 (1993).

[6] J. Masoliver and K. G. Wang, Phys. Rev. E 51, 2987 (1995).

[7] K. G. Wang and C. W. Lung, Phys. Lett. 151, 119 (1990).

[8] K. G. Wang, Phys. Rev. A 45, 833 (1992).

[9] R. Muralidhar, D. Ramkrishna, H. Nakanishi, and D. Jacobs, Physica A 167, 539 (1990).

[10] K. Lindenberg and B. J. West, The Nonequilibrium Statistical Mechanics of Open and Closed Systems (VCH, New York, 1990).
[11] R. Kubo, M. Toda, and N. Hashitsume, Statistical Physics II, Nonequilibrium Statistical Mechanics (Springer-Verlag, Berlin, 1985).

[12] R. M. Mazo, in Stochastic Processes in Nonequilibrium Systems, edited by L. Garrido, P. Seglar, and P. J. Shepherd (Springer-Verlag, Berlin, 1978).

[13] R. L. Stratonovich, Topics in the Theory of Random Noise (Gordon and Breach, New York, 1963), Vol. 1.

[14] W. Feller, An Introduction to Probability Theory and its Applications (Wiley, New York, 1971), Vol. II.

[15] N. Bleistein and R. A. Handelsman, Asymptotic Expansions of Integrals (Dover, New York, 1986). 
[16] R. A. Handelsman and J. S. Lew, SIAM J. Math. Anal. 5, 425 (1974).

[17] D. V. Widder, The Laplace Transform (Princeton University Press, Princeton, 1946).
[18] R. Muralidhar, D. Jacobs, D. Ramkrishna, and H. Nakanishi, Phys. Rev. A 43, 6503 (1991).

[19] J. Klafter, A. Blumen, G. Zumofen, and M.F. Shlesinger, Physica A 168, 637 (1990). 